EPJ Web of Conferences 81, 06006 (2014)

DOI: $10.1051 /$ epjconf/ 20148106006

(C) Owned by the authors, published by EDP Sciences, 2014

\title{
Experimental Investigation of the Few-Nucleon Dynamics in Deuteron-Deuteron Collision at $160 \mathrm{MeV}$
}

\author{
G. Khatri ${ }^{1, a}$, K. Bodek ${ }^{1}$, I. Ciepał ${ }^{1}$, N. Kalantar-Nayestanaki ${ }^{2}$, St. Kistryn ${ }^{1}$, B. Kłos ${ }^{3}$, A. Kozela ${ }^{4}$, \\ A. Magiera ${ }^{1}$, I. Mazumdar ${ }^{5}$, J. Messchendorp ${ }^{2}$, W. Parol ${ }^{1}$, D. Rozpȩdzik ${ }^{1}$, I. Skwira-Chalot ${ }^{6}$, \\ E. Stephan ${ }^{3}$, A. Wrońska ${ }^{1}$, and J. Zejma ${ }^{1}$ \\ ${ }^{1}$ Institute of Physics, Jagiellonian University, 30-059 Kraków, Poland \\ ${ }^{2} \mathrm{KVI}$ - Center for Advanced Radiation Technology, Uni. of Groningen, Groningen, 9747 AA, The Netherlands \\ ${ }^{3}$ University of Silesia, 40-007 Katowice, Poland \\ ${ }^{4}$ Institute of Nuclear Physics PAN, 31-342 Kraków, Poland \\ ${ }^{5}$ Tata Institute of Fundamental Research, Mumbai 400 005, India \\ ${ }^{6}$ Faculty of Physics, University of Warsaw, Warsaw, Poland
}

\begin{abstract}
An experiment, with unpolarized deuteron beam of $160 \mathrm{MeV}$ impinging on liquid deuterium target, was carried out using BINA detector at KVI, in Groningen, the Netherlands. Data were collected for the purpose of obtaining high precision differential cross-section for the deuteron break-up reaction. The elastic scattering data were also collected alongside for the purpose of cross-section normalization. We present here a sample of the un-normalised cross-section for the three-body final state $(\mathrm{dd} \rightarrow \mathrm{dpn})$ reaction.
\end{abstract}

\section{Introduction}

Understanding the exact nature of the nuclear force is one of the long-standing questions in nuclear physics. Yukawa in 1935 proposed meson-exchange theory of two interacting nucleons and nowadays there exists a number of very advanced theoretical models originating from this idea, such as Nijm-I and -II, Argonne V18 or CDBonn. Three-nucleon (3N) systems can be considered as the simplest non-trivial environment to test the modern models, therefore they have been studied extensively in both, theoretical and experimental aspects. With the new and improved experimental facilities, the measurement of very subtle effects like the three-nucleon force (3NF) [1-4] has now become possible. Theoretical treatment of the $3 \mathrm{~N}$ systems dynamics have also progressed in parallel, resulting with well description of the most of $3 \mathrm{~N}$ data. However, discrepancies still exists, especially in the sector of tensor analyzing powers [5]. The four-nucleon $(4 \mathrm{~N})$ systems can be considered as the next step, both interesting and difficult due to complexity of the final states, variety of spin and isospin combinations etc. For such environment, the knowledge is scarce in both, the theoretical and the experimental domain. Recently certain progress has been made in the calculations for dd systems [6], but so far at the energies below the breakup-reaction threshold. The 3NF effects are expected to be enhanced in $4 \mathrm{~N}$ system what makes the investigations even more attractive. With the aim to create the basis for understanding of the $4 \mathrm{~N}$ dynamics and testing the theoretical calculations, new precise data measured in a wide phase-space region have been collected.

ae-mail: g.khatri@uj.edu.pl 


\section{Experiment}

The BINA detector is a $4 \pi$ apparatus designed for few-nucleon scattering experiments at intermediate energies. BINA is divided into two main parts, forward Wall $\left(\theta: 13^{\circ}-40^{\circ}\right)$ and backward Ball $\left(\theta: 40^{\circ}\right.$ $165^{\circ}$ ). The forward Wall consists of (a) multi-wire proportional chamber (for reconstruction of angles of the scattered charged particles), (b) 12 vertical thin plastic scintillator 'stripes', and (c) 10 horizontal thick plastic scintillator 'slabs'. The plastic stripes and slabs form $240 \Delta \mathrm{E}-\mathrm{E}$ telescopes for particle identification. The backward Ball is nearly spherically symmetric, and made up of 149 triangular phoswich detector elements. The Ball at the same time plays the role of the reaction chamber as well as the detector. A detailed information about the detector can be found in [7]. For the purpose of the experiment described in this work, an unpolarized beam of deuteron with an energy of $160 \mathrm{MeV}$ was provided from AGOR cyclotron and was impinged on liquid deuterium targets.

\section{Data analysis, Results and Discussion}

Data were collected and a preliminary presorting was performed. Parts of runs characterized with unstable beam current or problems in functioning of any system elements were carefully removed. Geometry cross-check of the Wall detector part as well as correction of beam-shift from the target center were performed with the help of $d p$ elastic scattering (collected during the experimental run directly preceding the $d d$ experiment, performed with the same beam settings), allowing very precise momentum reconstruction of the detected charged particles. The elastically-scattered particles were
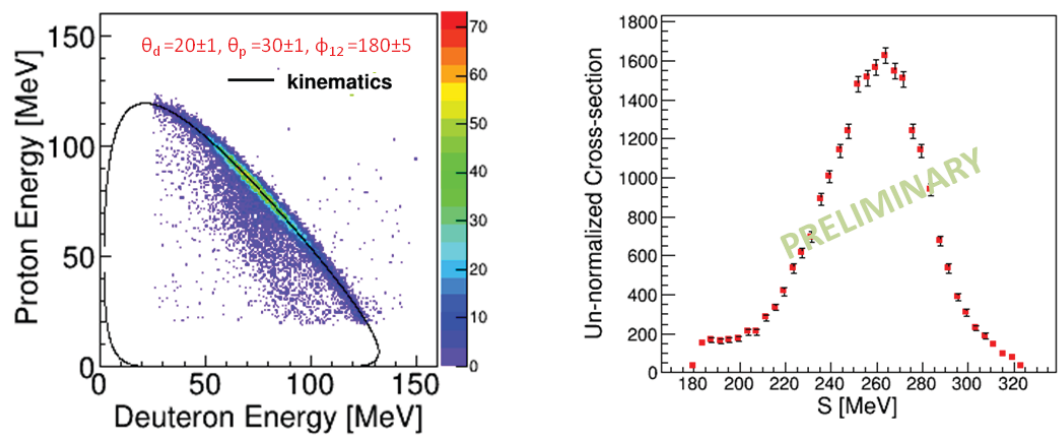

Figure 1. Preliminary results of breakup reaction $d d \rightarrow d p n$. Left panel: proton energy vs. deuteron energy spectrum for the angular configuration defined in the legend $\left(\theta_{d}, \theta_{p}\right.$ and $\phi_{12}$ represent the polar angles of the deuteron and proton and their relative azimuthal angle, respectively). The solid black line corresponds to the theoretical point-like geometry. Right panel: the unnormalised cross-section distribution for the same configuration.

also used for energy calibration purpose of the thick E-detector. In a first step particle identification (PID) based on a $\Delta \mathrm{E}-\mathrm{E}$ technique was performed. The basic assumptions of selection of the elastic scattering events were: (1) Coincidences of two charged particles in the detectors, and (2) Co-planarity $\left(\Delta \phi=180^{\circ}\right)$ of the double-track coincident events. For the purpose of calibration process, dedicated experimental runs were performed with energy degraders (aluminum plates of well-known thickness). The detection system response was simulated with the use of a GEANT4 tool. The simulated energy loss of the scattered particles in the E-detector were confronted with the experimental data, allowing to precisely calibrate the detector. Finally, a relation between the energy loss and energy at the reaction point was found. 
Having the proper calibration and PID implemented into analysis the three-body final state breakup channel ( $d d \rightarrow d p n$ ) was selected for further analysis. A set of deuteron-proton geometries, defined by their polar $\theta_{d}, \theta_{p}$ and relative azimuthal $\varphi_{12}=\varphi_{d}-\varphi_{p}$ angles were analysed. Energy of proton vs. energy of deuteron spectra (see Fig.1, left panel) were plotted for each configuration together with the result of the calculations for a point-like geometry kinematical curve. The breakup events grouped around the kinematics (in a range from- -12 to $+12 \mathrm{MeV}$ ) were divided into $\Delta S=8 \mathrm{MeV}$ and projected onto the calculated curve. For a given $S$-bin in the selected configuration, a number of the coincident proton-deuteron pairs was established. As a result a set of unnormalized cross-section distributions were obtained as a function of $S$-bin, see example in Fig.1, right panel. The statistical accuracy of the data is very promising, the analysis of systematic accuracy is under way.

The backard Ball was also under investigation with respect to geometry checks and energy calibration. Taking advantage of the axial symmetry of the Ball around the beam, it was possible to precisely calculate the angular information of the center of each crystal. Each event is, in fact, characterized by a number of clusters due to the problem with light-tightness of the crystal. The cluster consists then of a group of crystals or of a single crystal which registered signal in a given event. Therefore, the energy deposited in the detector is a sum of all energies deposited in crystals constituting the cluster. Besides energy, clusters are also characterized by a mean scattering angles, calculated as the weighted average of the angles corresponding to the geometrical centers of the cluster elements. Next step in the analysis is going to be the breakup cross-section normalization with the use of the $d d$ elastic scattering events. In order to achieve this goal, one needs to count the number of the $d d$ Wall-Ball coincidences. Applying kinematical restrictions of the elastic scattering one can expect to select $d-d$ pairs (imposing PID and energy cut on the elastically-scattered deuterons in the Wall detector).

The obtained precise experimental data in a wide phase-space region can serve as valid tool for verification of rigorous theoretical calculations which have been and are being developed. Before the rigorous calculation become available for $4 \mathrm{~N}$ system, the specific configurations, corresponding to quasi-free $d p$ scattering, with neutron as a spectator, can be compared with the predictions obtained in $3 \mathrm{~N}$ system [8].

\section{Acknowledgements}

We acknowledge support by the Foundation for Polish Science - MPD program, co-financed by the European Union within the European Regional Development Fund, Małopolskie Centrum Przedsiębiorczości - Project "Doctus - Małopolski fundusz stypendialny dla doktorantów" and the Polish 2013-2016 science founds as research Project No. 2012/05/E/ST2/02313. This work was supported by the European Commission within the Seventh Framework Programme through IA-ENSAR (contract no. RII3-CT-2010-262010).

\section{References}

[1] W. Glöckle et al., Phys. Rep. 274, 107 (1996).

[2] E. Epelbaum, Prog. Part. Nucl. Phys. 57, 654 (2006).

[3] N. Kalantar-Nayestanaki et al., Rep. Prog. Phys. 75 016301, 41pp (2012).

[4] E. Stephan et al., Eur. Phys. J. A 49: 36 (2013).

[5] E. Stephan et al., Phys. Rev. C 82, 014003 (2010).

[6] A. Deltuva. Few-Body Systems, Vol. 55, Issue 8-10, p621 (2014).

[7] St. Kistryn et al., Phys. Rev. C72, 044006 (2005).

[8] A. Ramazani-Moghaddam-Arani et al., Physics Letters B 725, 282-286 (2013). 